\title{
Superluminescence in Green Emission GaInN/GaN Quantum Well Structures under Pulsed Laser Excitation
}

Jayantha Senawiratne ${ }^{1,2}$, Stephanie Tomasulo ${ }^{2}$, Theeradetch Detchprohm ${ }^{1,2}$, Mingwei Zhu ${ }^{1,2}$, Yufeng $\mathrm{Li}^{1,2}$, Wei Zhao ${ }^{1,2}$, Yong Xia ${ }^{1,2}$, Zihui Zhang ${ }^{1,2}$, Peter Persans ${ }^{2}$, and Christian Wetzel ${ }^{1,2}$ ${ }^{1}$ Future Chips Constellation, Rensselaer Polytechnic Institute, Troy, NY, 12180

${ }^{2}$ Department of Physics, Applied Physics, and Astronomy, Rensselaer Polytechnic Institute, Troy, NY, 12180

\begin{abstract}
We report nonlinear optical investigation of green emission $\mathrm{GaInN} / \mathrm{GaN}$ multi-quantum structures grown along c- and $\mathrm{m}$-axes on sapphire and bulk GaN substrates, respectively. Under intense pulsed photo excitation, we observed strong superluminescence near the lasing condition in c-plane grown quantum well structures with full width at half maximum of $6 \mathrm{~nm}$. The superluminescence couples out of the edge of the sample in a mode pattern consistent with gain in a high mode of the waveguide. The wavelength of the superluminescence is $474 \mathrm{~nm}$. The threshold intensity of superluminescence was found to be $156 \mathrm{~kW} / \mathrm{cm}^{2}$. Increasing pump intensity leads to a strong photoluminescence blueshift as large as $380 \mathrm{meV}$ in samples grown along the c-axis on sapphire substrate, while under the same excitation conditions, the blue shift for the m-axis grown structure on bulk GaN substrate is less than $10 \mathrm{meV}$. The large emission blueshift is hereby attributed to the internal piezoelectric field in the c-axis grown structure. Its absence in the m-axis structure could enable low threshold current visible laser diodes.
\end{abstract}

\section{INTRODUCTION}

GaInN/GaN quantum well (QW) structures are the system of choice for UV and blue laser diodes (LDs) and also hold the biggest promise for green laser diodes [1,2]. The successful development of this material system, however, requires identification of the underling physical mechanisms for spontaneous and stimulated emission. In particular, for structures with high In content GaInN layers, large piezoelectric fields in c-axis oriented QWs are known to play a crucial role in the spontaneous emission process. Such a phenomenon also must be expected for the stimulated emission process [3,4]. By means of the quantum confined Stark effect (QCSE) in the fields of the strong piezoelectric polarization, electrons and holes tend to become separated in real space and so reduce the interband transition matrix element [4]. It is expected that even under high carrier injection conditions, the optical gain can detrimentally be affected in the consequence. On the other hand, potential fluctuations in the active layers are known to lead to a broadening of the optical emission band, further reducing the optical gain.

Here we report the optical properties of green emitting GaInN/GaN multi-QW (MQW) structures near the lasing conditions under pulsed and continuum $(\mathrm{cw})$ laser excitation. The effect of the polarization field on the optical properties is evaluated by comparing the emission properties of $\mathrm{GaInN} / \mathrm{GaN} \mathrm{QW}$ structures grown along the polar c-axis on c-plane sapphire substrate and along the non-polar $\mathrm{m}$-axis on $\mathrm{m}$-plane bulk GaN substrate. The frequently used expressions polar and non-polar growths are slightly misleading since they only refer to the conditions of an individual sample crystal, e.g., GaN. Heterostructure growth along the non-polar 
axis of GaN necessarily induces biaxial strain and the associated piezoelectric polarization. This is on top of the heterointerface induced pyroelectric components. Nevertheless, heterostructures grown along the m- or a-axes of $\mathrm{GaN}$ are promising and efficient approaches to vary and reduce piezoelectric polarization in GaInN/GaN QWs.

\section{EXPERIMENT}

GaInN/GaN MQW structures have been grown by metal organic vapor phase epitaxy (MOVPE) along the c-axis of sapphire (sample C) and along the $\mathrm{m}$-axis of bulk GaN (sample M) substrates. The MQW consists of five $\mathrm{Ga}_{1-x} \mathrm{In}_{x} \mathrm{~N} / \mathrm{GaN}$ QWs of $3 \mathrm{~nm}$ nominal well width separated by barriers of nominal width $11 \mathrm{~nm}$. From the fact that both structures were grown under identical MOVPE conditions, we assume the same alloy composition in all QWs $(x=0.15$ -0.20 ). More accurate values are not available at this time, in particular for the m-plane growth. For this recently developed growth orientation, new x-ray diffraction analysis methods have yet to be developed. The optical performance under pulsed and cw excitation was studied by photoluminescence (PL) spectroscopy in edge emission configuration using a $5 \mathrm{~cm}$ focal length spectrometer setup including a charged coupled device as the detector. High pump intensity measurements were carried out with a nitrogen laser $(\lambda=337.1 \mathrm{~nm})$ with $10 \mathrm{~ns}$ pulse width and maximum pulse energy of $0.1 \mathrm{~mJ}$. Low pump intensity measurements were carried out with a $\mathrm{HeCd} \mathrm{cw}$ laser of wavelength $325 \mathrm{~nm}$ and power $8 \mathrm{~mW}$. The optical pump intensity dependent PL measurement was performed by controlling pump beam intensity using calibrated glass slides as neutral density filters. The variable stripe length method [5] was used to measure the optical gain of the structure. Optical re-absorption measurements were performed by varying the separation of excitation spot location and the emission edge of the sample [6].

\section{DISCUSSION}

Fig. 1 shows the edge emission PL spectra of samples $\mathrm{C}$ and $\mathrm{M}$ plotted for several pump intensities under pulsed nitrogen laser excitation. The excitation stripe length in both cases was 5 $\mathrm{mm}$. The PL spectra were also measured under $\mathrm{cw}$ laser excitation at a low excitation density of $20 \mathrm{~W} / \mathrm{cm}^{2}$. While emission in the c-axis sample (C) peaks at $555 \mathrm{~nm}$ (Fig. 1a), emission in the $\mathrm{m}$ axis sample (M) shows a maximum at $492 \mathrm{~nm}$ (Fig. 1b). Apparently, despite the identical applied MOVPE conditions, a significantly shorter emission wavelength is observed in the structure of reduced polarization. While it cannot be excluded that this difference is due to different In incorporation properties of both growth surfaces despite the offered identical environment conditions, the more likely cause is the absence or reduction of polarization in the m-axis structure.

In the c-axis grown structure, PL under pulsed low excitation density appears at $494 \mathrm{~nm}$ and shifts to shorter wavelengths of $476 \mathrm{~nm}$ under high excitation density (See Fig. 1). In parallel, the full width at half maximum (FWHM) of the edge emission PL narrows from $82 \mathrm{~nm}$ to $6 \mathrm{~nm}$ and the far field pattern splits into two distinct maxima with enhancing its intensity exponentially (see Fig. 2(a)). These observations are clear indications of superluminescence. No such superluminescence behavior was observed in sample $M$ as pump intensity increases. Instead, in the absence of a far field pattern in sample M, PL intensity increases linearly with increasing pump intensity, while its FWHM stays greater than $20 \mathrm{~nm}$. The fact that we cannot 
observe superluminescence in sample $M$ is most likely due to the fact of the early stage of epitaxial growth optimization along the crystallographic m-axis.

The PL behavior in sample $\mathrm{C}$ is consistent with an optical amplification due to the stimulated emission. The threshold to superluminescence occurs at an external pump excitation density of $156 \mathrm{~kW} / \mathrm{cm}^{2}$ equivalent to $1.56 \mathrm{~mJ} / \mathrm{cm}^{2}$. A far field pattern consisting of two narrow beams propagating at a separation angle of 40 degree can be observed. This indicates that the highest gain waveguide mode is of a high spatial order of 5-10. This can be expected from the large asymmetry in our layer structure [7]. In fact, our calculations identify it as the $7^{\text {th }}$ order transverse electrical mode. It extends through the entire $\sim 5 \mu \mathrm{m}$ thick epitaxial layer surrounded by air and sapphire as upper and lower cladding layers, respectively.

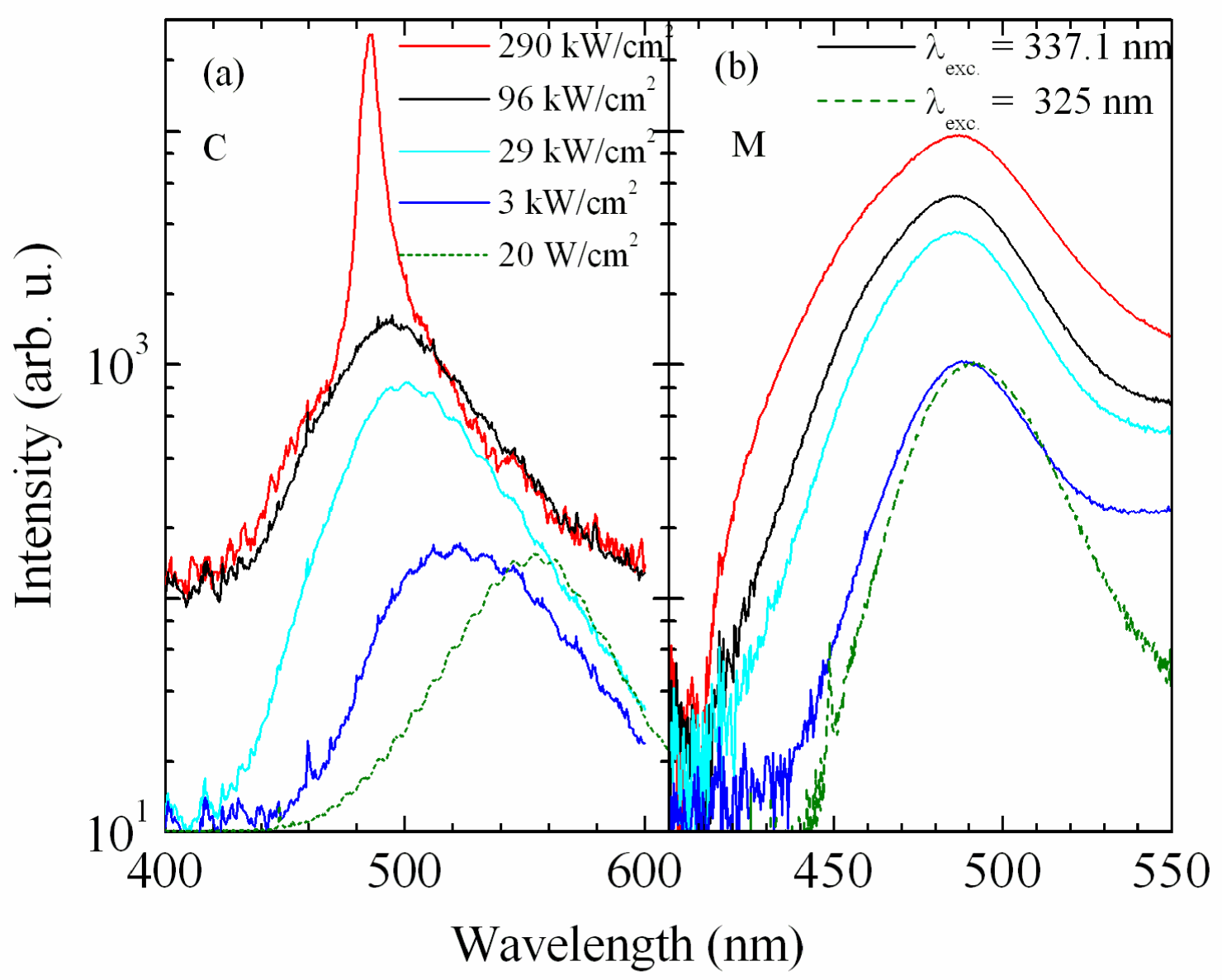

Figure 1 Room temperature PL spectra as a function of pump intensity for samples (a) C (cplane) and (b) M (m-plane). PL was collected in edge emission configuration. The solid and dashed lines represent PL under pulsed $(337.1 \mathrm{~nm})$ and $\mathrm{cw}(325 \mathrm{~nm})$ excitations, respectively.

Fig. 2 summarizes the PL peak wavelength, intensity, and FWHM of sample C plotted against pump intensity. Under high excitation density, a PL blueshift of about $82 \mathrm{~nm}(380 \mathrm{meV})$ was observed relative to the PL peak maxima of $555 \mathrm{~nm}$ under low intensity cw excitation. 
Furthermore, comparing different c-axis grown QW structures, we observed that the $I_{\text {exc-induced }}$ blueshift increases with the emission wavelength. The PL blueshift for the non-polar sample $\mathrm{M}$ measured under the same conditions is only a few nanometers $(10 \mathrm{meV})$. We attribute this relevant difference to the strong piezoelectric polarization across the QW that only acts in c-axis grown structure. This polarization shifts the emission peak to the lower energy side. Then, as the excitation pump intensity increases, the internal field may in part become screened by the injected carrier pairs and the emission line shifts towards a wavelength corresponding to the absence of piezoelectric polarization. The observation of a lager blueshift in samples with longer emission wavelength supports this interpretation, since the polarization dipole increases with the InN-fraction in the c-axis oriented QW.

Optical gain measurements were performed using the variable stripe length method under edge emission configuration. The samples were excited with a rectangular narrow laser stripe of dimension $5 \mathrm{~mm} \times 0.69 \mathrm{~mm}$ with intensity of $10 \mathrm{~kW}$ within the stripe. The laser power was measured externally, however, due to the reflection and scattering at the sample surface, the net energy that propagates into the MQW structure could be somewhat less than the given value. The PL intensity, integrated over the wavelength range from $400 \mathrm{~nm}$ to $550 \mathrm{~nm}$ is plotted against the stripe length for sample $\mathrm{C}$ in Fig. 3. During the gain measurement, the stripe length was varied from $0 \mathrm{~mm}$ to $5 \mathrm{~mm}$ in steps of $0.125 \mathrm{~mm}$ using a traveling knife edge to control the laser stripe length.
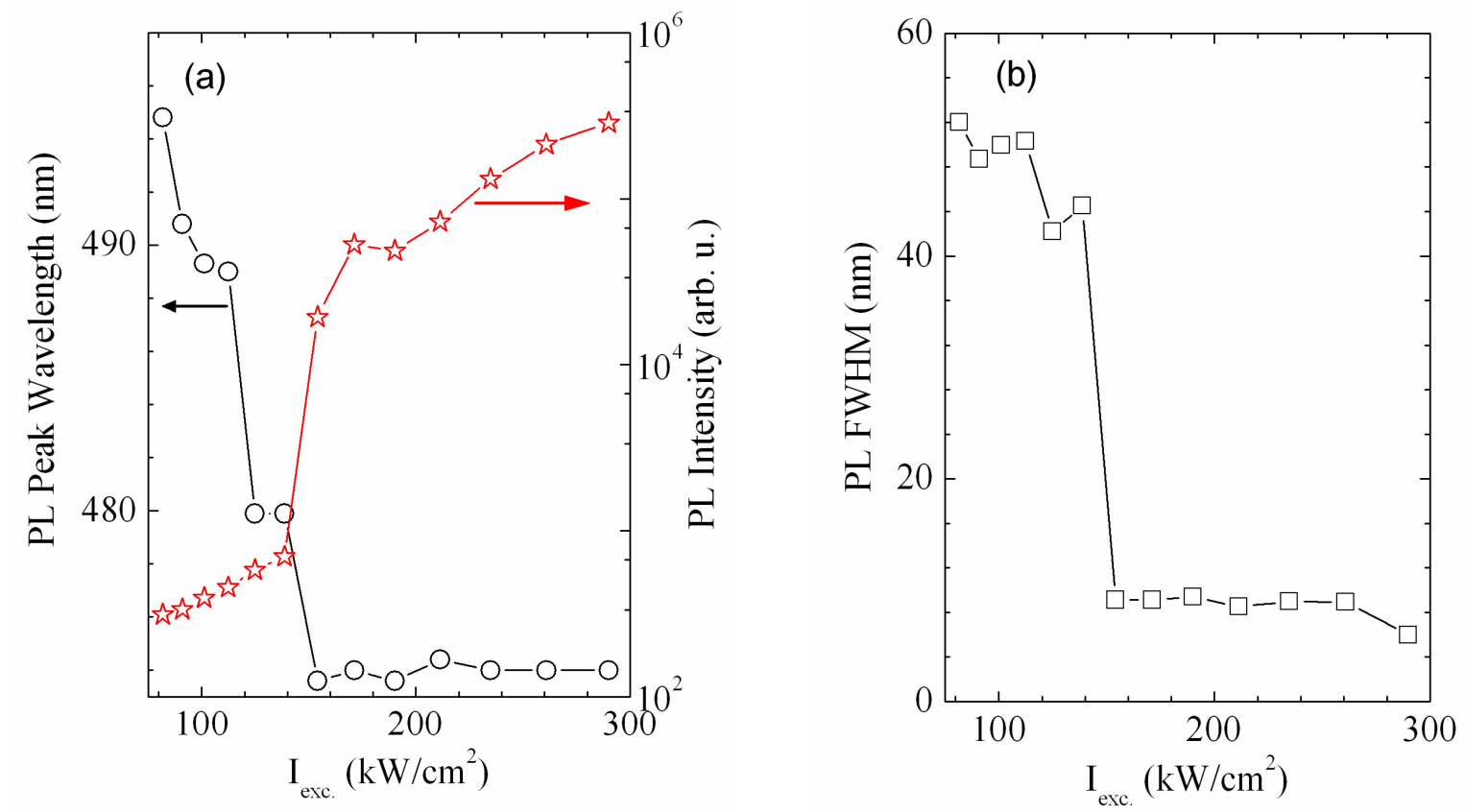

Figure 2 PL (a) peak position (black), intensity (red), and (b) FWHM as a function of pump $I_{\mathrm{exc}}$ for sample $\mathrm{C}$.

For small stripe length, the integrated PL intensity varies almost linearly with the stripe length. When the stripe length exceeds $3.75 \mathrm{~mm}$, the PL intensity begins to increase 
exponentially with increasing length. At the same time, the PL peak width decreases dramatically as the output in the high intensity PL grows exponential with length. Moreover, the output on either side of the peak only grows slowly, as the stripe length exceeds $3.75 \mathrm{~mm}$. The net gain of the integrated output is determined from the slope of the log of the PL intensity versus stripe length $(>3.75 \mathrm{~mm})$. The net wavelength-integrated superluminescent gain for (polar) sample $\mathrm{C}$ is thus $20 \mathrm{~cm}^{-1}$. This value at $474 \mathrm{~nm}$ is lower than the reported optical gain values in similar structures at $425 \mathrm{~nm}[8,9]$. However, the superluminescence in our sample occurs at significantly longer wavelengths. It has been reported that the oscillator strength between electron and hole in the QW weakens as the piezoelectric field increases, thus reduces the optical gain [10]. Therefore, it is possible that the higher InN-fraction associated with the larger polarization field these longer wavelength samples results in lower optical gain due to the reduced oscillator strength.

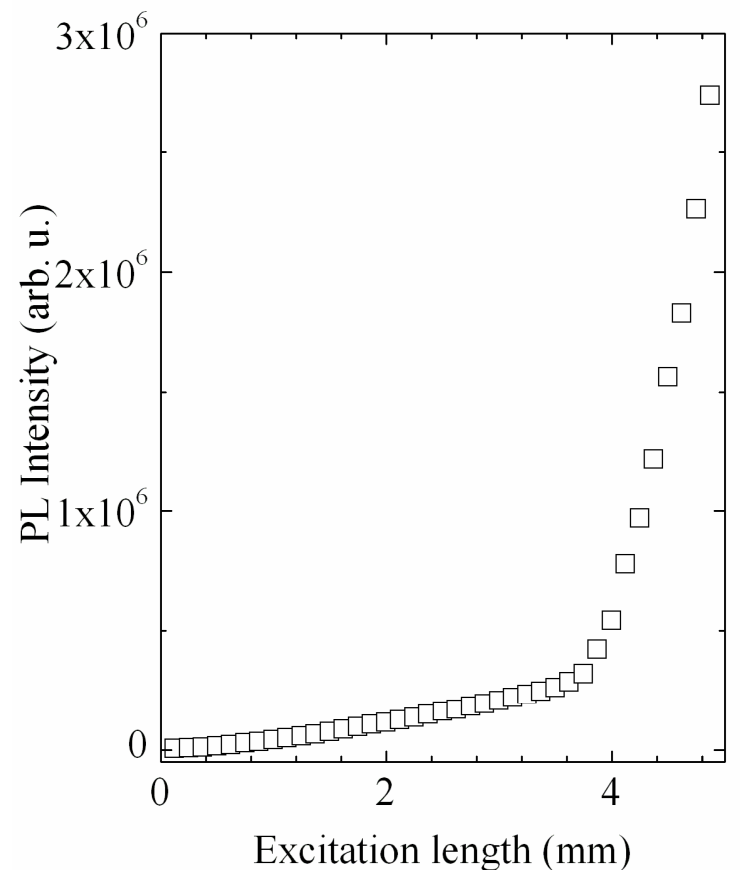

Figure 3 Intensity of the PL emission as a function of stripe length for sample $C$.

Superluminescence occurs when the stripe length reaches $3.75 \mathrm{~mm}$. Beyond this, the PL intensity increases exponentially with stripe length.

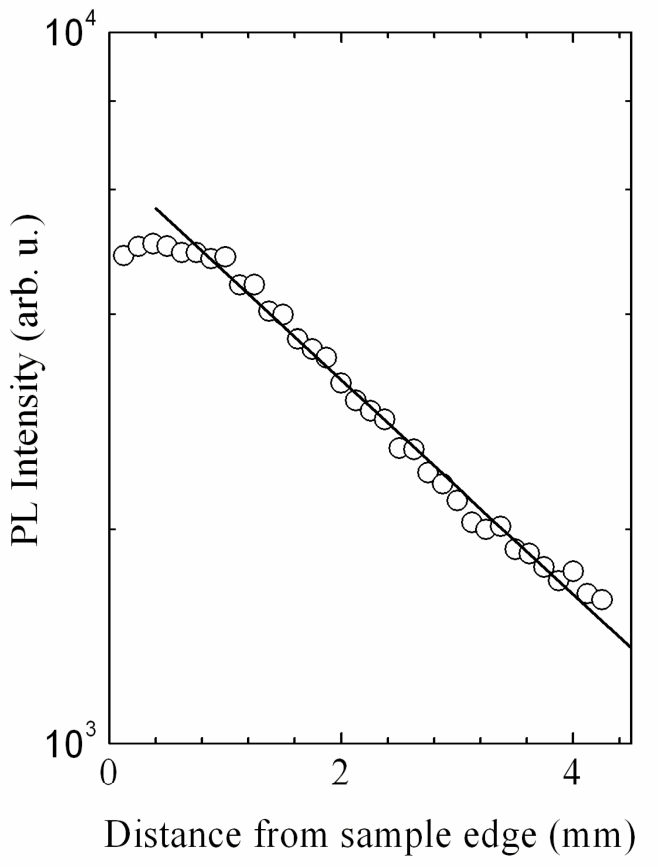

Figure 4 Optical absorption measurement was performed by shifting the excitation spot. The solid line is a fit to the experimental data of sample $\mathrm{C}$ (open circles).

The measured optical gain is strongly affected by optical re-absorption known as absorption loss. The absorption loss can be estimated by using a small excitation beam spot under conditions of spontaneous luminescence and moving the spot across the sample, increasing the travel length of the luminescence through the sample to the emission edge. The result of the measurement is shown in Fig. 4 and indicates that this absorption loss is about $2.3-6.0 \mathrm{~cm}^{-1}$. In the literature, similar absorption loss values have been reported for GaInN/GaN epitaxial layer structures with similar defect density $\left(\sim 10^{8} \mathrm{~cm}^{-2}\right)[11,12]$. This is an extremely low value. 


\section{CONCLUSIONS}

In conclusion, we have investigated the optical properties of GaInN/GaN QW structures grown along $\mathrm{c}$ - and $\mathrm{m}$-axes of $\mathrm{GaN}$ under high photon excitation density and observed strong nonlinear optical behavior in the sample grown along the c-axis. We observed strong superluminescent emission at $474 \mathrm{~nm}$. The pump intensity threshold for $10 \mathrm{~ns}$ pump pulses is $156 \mathrm{~kW} / \mathrm{cm}^{2}$ (or $1.56 \mathrm{~mJ} / \mathrm{cm}^{2}$ ). The PL peak wavelength for this polar sample shifts from $555 \mathrm{~nm}$ up to $474 \mathrm{~nm}$ as the excited carrier density increases from $82 \mathrm{~kW} / \mathrm{cm}^{2}$ to $290 \mathrm{~kW} / \mathrm{cm}^{2}$. The optical gain was found to be $20 \mathrm{~cm}^{-1}$, which is somewhat lower than literature data for shorter wavelength emitters. For the development of laser diodes, the large blue shift is considered a hurdle towards low threshold currents. While still no superluminescence could be observed in the non-polar m-axis structure, the absence of the blue shift makes this system the more likely choice for low threshold current visible laser diodes..

\section{ACKNOWLEDGMENTS}

This work was supported by a DOE/NETL Solid-State Lighting Contract of Directed Research under DE-FC26-06NT42860 and the DARPA VIGIL program through the United States Air Force AFRL/SNH under FA8718-08-C-0004.

\section{REFERENCES}

1. I. Akasaki, in Nitride Semiconductors, Mat. Res. Soc. Symp. Proc. Vol. 482 (1998 Materials Research Society). p. 1.

2. C. Wetzel, T. Salagaj, T. Detchprohm, P. Li, J. S. Nelson, Appl. Phys. Lett. 85, 866 (2004). 3. K. Kojima, M. Funato, Y. Kawakami, S. Nagahama, T. Mukai, H. Braun, and U. T. Schwarz, Appl. Phys. Lett. 89, 241127 (2006).

4. C. Wetzel, H. Amano, and I. Akasaki, Jpn. J. Appl. Phys. 39, 2325 (2000).

5. K. L. Shaklee, R. E. Nahory, and R. F. Leheny, J. Lumin, 7, 284 (1973).

6. A. Moritz, R. Wirth, C. Geng, F. Scholz, and A. Hangleiter, Appl. Phys. Lett. 68, 1217 (1996).

7. D. Hofstetter, D. P. Bour, R. L. Thornton, and N. M. Johnson, Appl. Phys. Lett. 70, 1650 (1997).

8. T. Swietlik, P. Perlin, T. Suski, M. Leszczynski, R. Czernecki, I. Grzegory, and S. Porowski, Phys. stat. sol. (c) 4, 82 (2007).

9. Y. Cho, T. J. Schmidt, S. Bidnyk, G. H. Gainer, J. J. Song, S. Keller, U. K. Mishra, and S. P. Denbaars, Phys. Rev. B 61, 7571 (2000).

10. J. S. Im, H. Kollmer, J. Off, A. Sohmer, F. Scholz, and A. Hangleiter, Phys. Rev. B 57, R9435 (1998).

11. M. Rowe, M. Vehse, P. Michler, J. Gutowski, S. Heppel, and A. Hangleiter, Phys. stat. sol.(c) 0,(6), 1860 (2003).

12. G. Frankowsky, F. Steuber, V. Harle, F. Scholz, and A. Hangleiter, Appl. Phys. Lett. 68, 3746 (1996). 